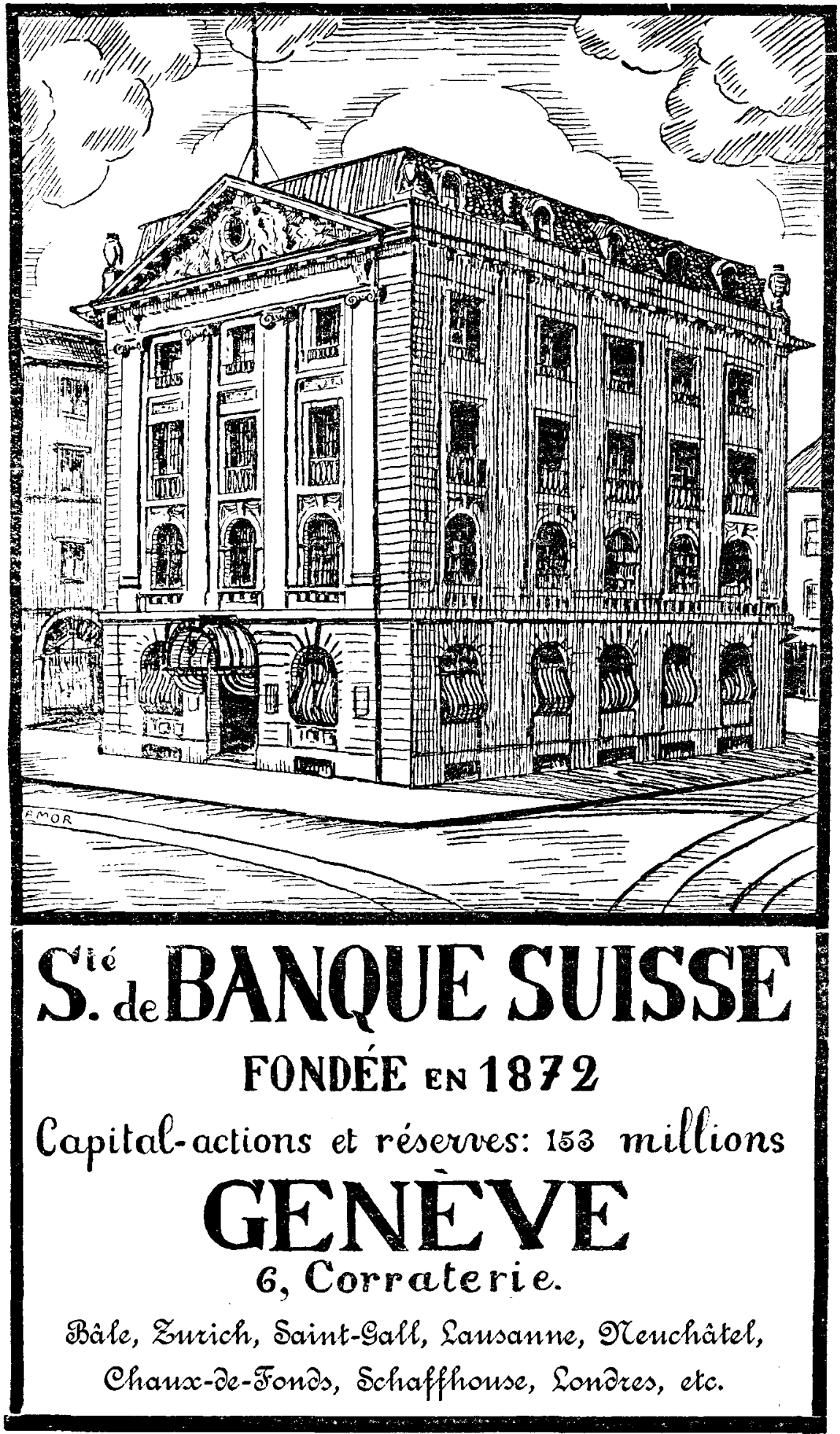




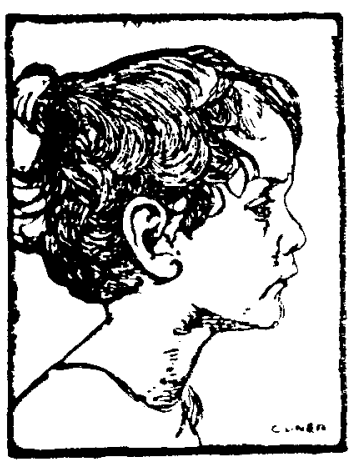

\section{"Pro Juventute”}

Revue mensuelle illustrée pour la protection de la jeunesse.

Cette revue, qui publie des articles en français, allemand et italien et est dirigée dans un esprit de parfaite neutralité religieuse et politique, se propose de devenir de plus en plus le centre de ralliement de toutes les cuvres pour la protection de la jeunesse en Suisse les auvres similaires de l'étranger.

et de nouer des relations suivies avec

Sa lecture présente donc un intérêt très réel pour toutes les personnes qui comprennent l'importance primordiale qu'offre à notre époque la formation d'une jeunesse forte et saine.

Prix de l'abonnement: frs. 7.-. par an.

On s'abonne a tous les bureaux de poste suisses ainsi qu'au Secrétariat central Pro Juventute, Untere Zäune II, Zurich, I. Pour les annonces, s'adresser au Secrétariat central.

Compte de chèques postaux, VIII 3 Ioo, Zurich.

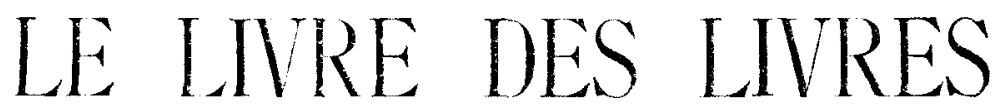

Nous sommes heureux de signaler, à ceux de nos lecteurs qui ne la connaitraient pas, cette anthologie critique mensuelle des nouveaux ouvrages littéraires, dont chaque numéro, qui contient la Critique, l'Analyse d'importants Extraits (texte et illustrations) des volumes récemment parus, permet to d'être rapidement et bien au courant des dernicres productions ; $2^{\circ}$ de faire soll choix en connaissance de cause.

ine telle revue cst particulièrement utile à notre époque où les livres coûtent si cher!

Abonnement : France, un an, $14 \mathrm{fr}$. ; six mois, $7 \mathrm{fr}, 50$; trois mois, $4 \mathrm{fr}$. - Etranger, un an, $16 \mathrm{fr}$.; six mois, $8 \mathrm{fr} .50$; trois mois, $4 \mathrm{fr} .50$. - Le numéro: France, $1 \mathrm{fr} .50$; Etranger, $1 \mathrm{fr} .70$.

Unc augmentation de prix est à craindre.

Il reste quelques rares collections des $\mathbf{2}$ premiers numéros parus, qui sont expédiés en France, contre $x_{4} \mathrm{fr}$. et à l'étranger contre $\mathrm{I} 6 \mathrm{fr}$.

Adresser la correspondance au directeur, M. Gaston Moussé, 3, rue du Marché des Patriarches, Paris (Vre). 


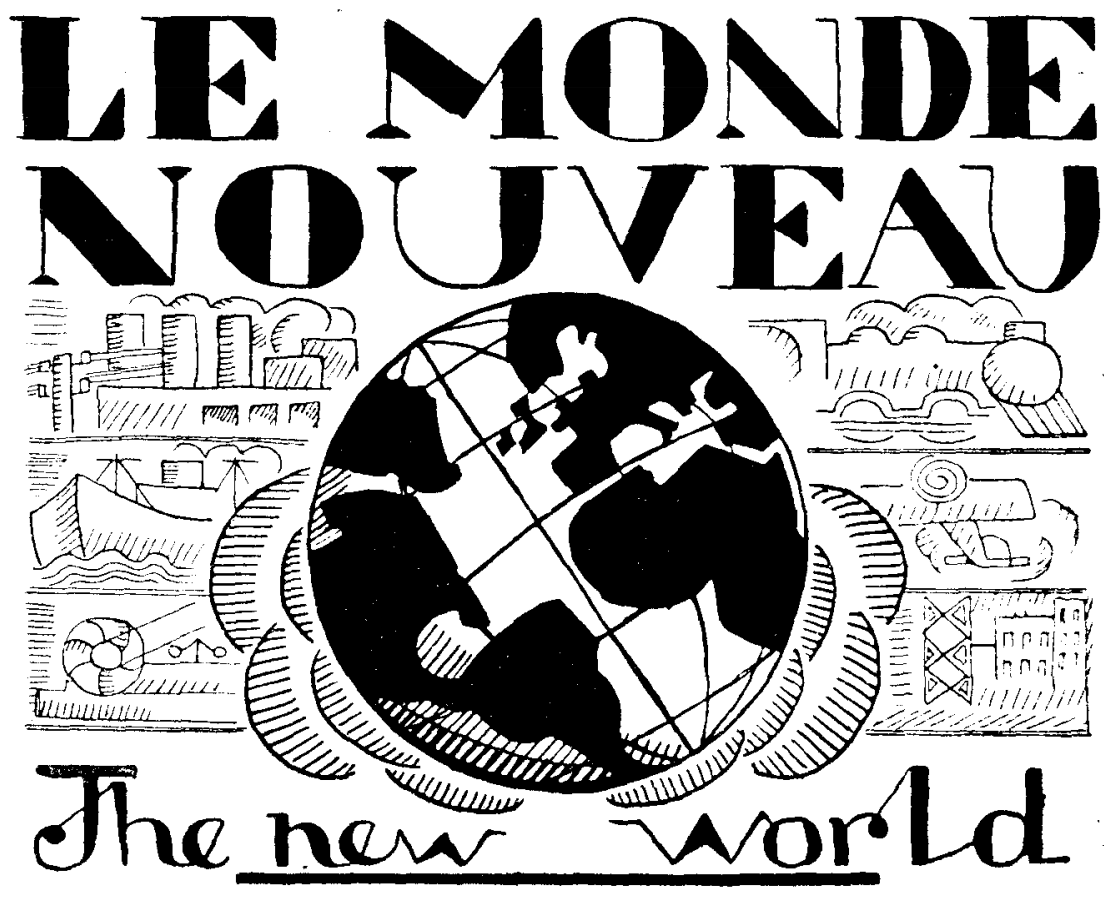

\section{REVUE MENSUELLE INTERNATIONALE} paraissant le 20 de chaque mois

Rédaction et Administration : $4^{2}$, boulevard Raspail, PARIS $-7^{\mathrm{e}}$ Tél. Fleurus 27-65.

Publicité : 20, rue Boissy d'Anglas, PARIS - 8. Tél. Elysée 61-18. Bureaux à Londres : 8 Stone Buildings, W. C. 2, Tel. Musuel 6398 .

ABONNEMENTS : Edition française. Etranger Edition anglaise

Directeur: Rédacteur en chef :

Ebed, VAN DER VLUGT Gustave-Louis TAUTAIN

40 francs par an
50 "
$45 \mathrm{Sh}$.

Secrétaire général: Adrien LE CORBEAU

DANS CHAQUE NUMÉRO:

Politique. - Sociologie. - Sciences financières et économiques. Lifférature et Beaux Srts. - Revue du mois. - Nos colonies

Industrie et Commerce. - Importations et Exportations. Nos Débouchés. - Nos besoins.

Une double édition de langue anglaise et de langue française parait simultanément à PARIS, à LONDRES et à NEW-YORK.

150 pages de texte, format in-Bo carré. 


\section{A VENDRE}

au profit de

l'Union internationale de secours aux enfants

UN

\section{UN ALBUM DE TIMBRES-POSTE}

constitué spécialement pour l'Union internationale de secours aux enfants par les membres de l'Union philathélique de Genève.

Cet album contient plus de 2000 timbres d'Europe d'une valeur de plus de 5000 francs français d'après le catalogue Yvert et Tellier 1921. Les principales pièces sont les suivantes:

\begin{tabular}{|c|c|c|c|c|c|}
\hline Alsace & catalogule & Yvert e & Tellier 1921 & No & $7^{b}$ \\
\hline Bavière & 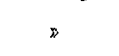 & $"$ & $D \quad$ & " & 7,14 \\
\hline Belgique & » & $"$ & $»$ & $\triangleright$ & 1,2 \\
\hline Brunswick & » & » & » & $\triangleright$ & 4 \\
\hline Sicile & » & » & $»$ & » & 19,20 \\
\hline Espagne & 》 & » & 》 & $"$ & 62 \\
\hline Finlande & » & $»$ & » & » & 48 \\
\hline France & > & $"$ & $»$ & » & 5 en paire, 40,47 \\
\hline Hanovre & $\triangleright$ & $»$ & » & * & 8 \\
\hline Levant Russe & 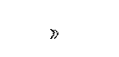 & » & » & » & $1^{\mathrm{a}}, 173,174$ \\
\hline Suisse & $"$ & » & $"$ & 》 & 2 \\
\hline
\end{tabular}

Tous les pays sont bien représentés. L'ensemble conviendrait parfaitement à un amateur désireux de posséder une collection embryonnaire en vue de la continuer.

\section{Prix : 1500 francs argent suisse.}

Ecrire à M. le Trésorier de l'U. I. S. E., 4, rue Massot, Genève. 
VIENT DE PARAITRE :

\title{
L'Union internationale de Secours aux Enfants
}

\author{
Genève, 4, rue Massot, 1920. \\ In- $8,4^{8}$ et roo $\mathrm{p}$.
}

Album contenant les portraits de membres du Comité d'honneur de l'Union, les autographes de huit membres du Comité d'honneur, les listes des Comités collecteurs affiliés à l'Union en Allemagne, Arménie, Finlande, France, Grande-Bretagne, Italie, Pays-Bas, Suède, Suisse, Turquie et 175 photographies de groupes d'enfants éprouvés des pays suivants : Albanie, Allemagne, Albanie, Autriche, Belgique, Esthonie, France, Hongric, Italie, Lettonie, Pologne, Roumanie, Russie, Serbie (S. H. S.), Turquie.

Prix : 5 frs. (argent suisse).

\section{BULLETIN DE L'UNION INTERNATIO- NALE DE SECOURS AUX ENFANTS}

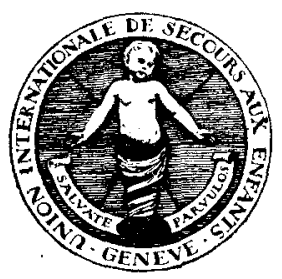

Save the Children Fund: International Union Internationale Vereinigung für Kinderhilfe Unione Internationale per la salvezza dei bambini

sous le patronage du

COMITÉ INTERNATIONAL DE LA CROIX-ROUGE

Rédaction et Administration :

4, Rue Massot, Genève (Suisse)

Parait sur I 2 ou 16 pages in-8, les 10 , 20 et 30 de chaque mois. A la plupart des numéros est adjoint un supplément illustré de 4 pages en photogravure. Prix de l'abonnement 9 frs. par an. Sous la forme la plus concise ce bulletin trimestriel relate les faits les plus marquants du mouvement international du secours aux enfants des pays éprouvés par la guerre, collectes catholiques, protestantes, orthodoxes, israélites, collectes socialistes, etc., allocations aux cuvres de secours aux enfants, rapports sur l'activité de ces cuvres tant américaines qu'européennes, renseignements impartiaux sur la misère des enfants, en Europe et dans le Proche Orient.

L'Union internationale de secours aux enfants n'a aucun caractère politique ni confessionnel, elle se borne à recueillir et à répartir des fonds et s'abstient de toute action directe. 


\section{LA REVUE DE GENÈVE}

PARAIT TOUS LES MOIS, SUR

I6O PAGES IN-8 $8^{\circ}$ AU MINIMUM

Internationale, mais non internationaliste, intersociale mais non socialiste, la Revue de Genève est une revue de liaison intellectuelle et de documentation originale. Seule, elle joue ce rôle nécessaire à une époque qui, après tant de bouleversements, réclame qu'on reconstruise. Dans ce chaos 'douloureux, elle cherche à retrouver les lignes directrices; elle contribue à préserver notre civilisation soumise à tant de menaces.

Chacun de ses numéros se divise en trois rubriques. Une première partie contient des cuvres d'imagination, des études de caractère général, des essais politiques, historiques, critiques. La seconde partie donne des "Chroniques nationales", lesquelles sont rédigées par des ressortissants des pays dont elles traitent. Tirant la philosophie des événements, ces chroniques fournissent des vues d'ensemble et donnent un tableau comparé, une image synthétique du monde moderne.

A ces "chroniques nationales" succède une "chronique internationale " consacrée à retracer les efforts des peuples non plus pour s'affirmer, mais pour s'entendre les uns les autres. On y trouve l'analyse des grands problèmes qui se posent à toutes les nations en commun, la libre discussion des diverses institutions universelles dont Genève est le siège, le compte rendu de l'activité internationale dans le monde entier.

La Revue de Genève compte parmi ses collaborateurs: MM. Maurice Barrès, René Boylesve, Georges Duhamel, Edouard Estaunié, Georges Eekhoud, Elie Faure, Daniel Halévy, Emile Henriot, Edmond Jaloux, Camille Mauclair, Pierre Mille, Edmond Pilon, Henri de Régnier, Jean Richard-Bloch, Jules Romains, André Suarès, J. J. Tharaud, Albert Thibaudet, Jean-Louis Vaudoyer, Benedetto Croce, G. Ferrero, Piero Jahier, G. Papini, Vilfredo Pareto, G. Prezzolini, Joseph Conrad, Georges Moore, Bernard Shaw, J. Zangwill, Arnold Bennett, John Erskine, Charles Macfarland, E. Curtius, F. W. Förster, Freud, H. Kessler, Heinrich Mann, W. Rathenau, J. Redlich, Maxime Gorki, Kouprine, Remisov, Sologoub, J. Bojer, Geijerstmamm, Per Hellstroem, Jules Andrassy, Fr. Riedl, J. de Voïnovitch, Andreades, N. Jorga, Ad. Salazar, Alfonso Reyes, Miguel Unamuno, Graça Aranha, etc., ainsi que les écrivains suisses les plus importants.

On s'abonne à la Revue de Genève, 46, rue du Stand, Genève (Editions Sonor), et chez les principaux libraires.

\begin{tabular}{|c|c|c|c|c|c|}
\hline Suisse.... . . . . . . . . & Fr. & 36.- - & Fr. & 19.- & Fr. \\
\hline Etranger (argent suisse)... & $"$ & 44. - & $"$ & 23. - & $"$ \\
\hline français)............... & $"$ & 60. & $"$ & $32 .-$ & $"$ \\
\hline
\end{tabular}




\title{
JOURNAL OF COMPARATIVE LEGISLATION
}

\author{
and \\ INTERNATIONAL LAW
}

April $192 \mathrm{I}$.

Issued to Subscribers only.

\author{
Review of the Legislation of the British Dominions During \\ the Year, I9I9.
}

"None of the services which the Society of Comparative Legislation renders the profession and the Empire is more valuable than the publication in its Journal of an annual review of the legislation of the British Dominions and of the principal foreign countries."

"These periodical reviews of legislation in the Journal of Comparative Legislation never fail to show how legislatures in different parts of the world deal simultaneously with the same problems." Sir Courtenay Ilbert rightly claims that the review constitutes a repertory of recent legislative experiments unparalleled in range and extent, and abounding in instruction and suggestion to those who, in different parts of the world, are concerned or interested in legislation. Not the least of its merits is that it sometimes indicates what ought not to be imitated or repeated."

Lares Journal.

The following reprints of articles from the Society's Journal may be obtained at One Shilling each .

Death Certification, by Prof. Glaister. Land Settlement of exservice men, by A. J. Hannan. Federal Constitutions within the Empire, by Viscount Haldane. Adoption, by Sir Alfred Hopkinson. Breach of promise of marriage, by Edward Manson. Criminal Procedure in France \& England, by Leon Montluc. The new Constitution of China, by H. B. Morse. Recent municipal experiments in U. S. A., by prof. Wm. Bennett Munro. Police Power in American constitutional Law, by Prof. T. Reed Powell. Women as practitioners of Law, by the Hon. Mr. Justice Riddell. The Relations Between the English law and the Personal law of Indians in England, with special reference to the masriage law, by Sir Fred. Robertson, Entities and veal and artificial persons, by W. E. Singleton, $A$ new basis for industrial Corporations, by G. R. Stirling Taylor. Proportional Representation in modern Legislation, by J. Fischer Williams, C. B. E.

Annual Subscription, One Guinea.

Society of Comparative Legislation.

I, Elm Court, Temple, London E. C. 4. 
CHINE. - Comité central de la Société chinoise de la Croix-Rouge, Pékin et 26 Kinkiang Road, Shanghaï.

CUBA. - Société nationale cubaine de la Croix-Rouge, rue O'Reilly, 6, La Havane.

DANEMARK. - Société danoise de la Croix-Rouge, Ved Stranden, 2, Copenhague, $\mathrm{K}$.

ESPAGNE. - Société espagnole de la Croix-Rouge, Atocha, 65, Madrid.

ESTHONIE. - Société de la Croix-Rouge esthonienne, Réval.

ETATS-UNIS. - Central Committee of the American National Red Cross, Washington, D. C.

FINLANDE. - Direction centrale de la Croix-Rouge de Finlande, Helsingfors.

FRANCE. - Comité central de la Croix-Rouge française, rue François Ier, 21, Paris, $8^{\text {me }}$.

GRANDE-BRETAGNE. - British Rëd Cross Society, 19, Berkeley Street, Londres, W. I.

GRĖCE. - Croix-Rouge hellénique, Athènes.

HESSE. - Société hessoise de la Croix-Rouge, Darmstadt.

HONGRIE. - Société hongroise de la Croix-Rouge, Andrassy-ut, 8, Budapest, VI.

INDES ANGLAISES. - Société de la Croix-Rouge des Indes anglaises, Hoal-Quarters, Delhi.

INDES NEERLANDAISES. - Société de la Croix-liouge des Indes néerlandaises, Batania.

ITALIE. - Croix-Rouge italienne, via Toscana, 10, Rome.

JAPON. - Société japonaise de la Croix-Rouge, Tokio.

LATVIA. - Croix-Rouge lettone, Latvia.

LUXEMBOURG. - Société luxembourgeoise de la Croix-Rouge, Luxembourg

MEXIQUE. - Comité central de la Société mexicaine de la Croix-Rouge, 2 a de San Geronimo, 14, Mexico, D. F.

NORVÉGE. - - Société norvégienne de Ia Croix-Rouge, Akersgaten, 44, Christiania.

NOUVELLE-ZÉLANDE. -- Croix-Pouge de la Nouvelle-Zélande, P. O. Box 969, Wellinglon, N. Z.

PAYS-BAS. - Comité Supérieur de la Croix-Rouge néerlandaise, Princessegracht, 29, La Haye.

PÉROU. - Société péruvienne de la Croix-Rouge, Lima.

POLOGNE. - Société polonaise de la Croix-Pouge, Mazowiecka, 9, Varsovie.

PORTUGAL. - Société portugaise de la Croix-Rouge, Lisbonne.

PRUSSE. - Comité central de l'Association prussienne de la Croix-Rouge, am Karlsbad, 23, Berlin, IV. 35.

ROUMANIE. - Société nationale de la Croix-Pouge de Roumanie, Academiei, 20, Bucarest.

RUSSIE. -

SAXE. - Société sasonne de la Croix-Rouge, Dresde.

SERBIE. - Société serbe de la Croix-Rouge, Simina ulica, 21, Belgrade.

SIAM. - Société siamoise de la Croix-Rouge, Bangkok.

SUĖDE. - Comité central de la Société suédoise de la Croix-Rouge, Artillerigatan, 6, Slockholm, 5 .

SUISSE. - Croix-Rouge suisse, rue des Cygnes, 9, Berne.

TCHÉCOSLOVAQUIE. - Société tchécoslovaque de la Croix-Pouge, Prague.

TURQUIE. - Comité central de la Société du Croissant-Rouge ottoman, Mahmoudié Djaddessi, Constantinople.

URUGUAY. - Comité central de la Croix-Rouge uruguayenne, calle Colon, 1382, Montevideo.

VENEZUELA. - Senor Presidente de la Sociedad Venezolana de la Cruz-Roja, Este 6, No 55, Caracas.

WURTEMBERG. - Société wurtembergeoise de la Croix-Rouge, Stutlgart. 


\section{Sommaire du Bulletin}

T. LII, N• 225

Comité international. - Accord entre le Comité international de la Croix-Rouge et la Ligue des Sociétés de la Croix-Rouge, 487. Missions et délégations, 488. En Allemagne, 490. En Esthonie, 494. En Hongrie, 498. En Pologne, 498. .En Russie, 5oo. En Sibérie, 50o. En Turquie, 501. En Yougoslavie, 507. - Rapatriement des prisonniers de guerre, internés et réfugiés (carte), $502-503$.

Ligue. - Secrétaire général, 5 I 2.

Australie. - Bulletin de la Croix-Rouge australienne, 5 I 2.

Chill. - Réorganisation de la Croix-Rouge du Chili et statuts nouveaux, 513. - Publications, 528.

Etats-Unis. - Le programme de paix de la Croix-Rouge américaine, 528.

France. - Activité de la Croix-Rouge en temps de paix, 53o. - Abus de la Croix-Rouge, 532. - Action de la Croix-Rouge française en Autriche, 534 .

Grèce. -- Composition du Comité central, 535.

Japon. - Secours aux affamés de Chine, 536. - La Croix-Rouge japonaise et les orphelins polonais, 537. - Chronique de la CroixRouge japonaise, 537 .

Portugal. - L'enregistrement des marques de commerce à croix rouge, 538. - Organisation générale de la Croix-Rouge portugaise (Décret du I9 novembre I920), 542. - Règlement du Corps actif de la Société portugaise de la Croix-Rouge (approuvé par décret du I9 août 1919), 564 .

Siam. - Composition du Conseil de la Croix-Rouge du Siam, 570.

Suède. - Assemblée générale de la Croix-Rouge suédoise, 570 .

Suisse. - Publications, 571 .

Uruguay. - Loi du i6 juillet igı 9 concernant l'usage du nom et du signe de la Croix-Rouge.

La Revue internationale de la Croix-Rouge, Bulletin international des Sociétés de la Croix-Rouge parait le 15 de chaque mois.

Prix, franco, un an : Fr. 20.- Le numéro: Fr. 2.Années I9I9 et 1920 (rares): Fr. 30.-

Administration : Promenade du Pin, 1, Genève.

Pour la publicité de la Revue, s'adresser à Orell Fussli-Publicité, rue de la Croix-d'Or, 2-4, Genève, et à ses succursales: Lausanne, Neuchâtel, Barne, Bâle, Soleure, Aạrau, St-Gall, Zurich.

Impqrimerie du Journal de Genève, sue Gïnéral-Dufous. 